\title{
Article \\ On a Class of Differential Variational Inequalities in Infinite-Dimensional Spaces
}

\section{Savin Treanţă}

Citation: Treanţă, S. On a Class of Differential Variational Inequalities in Infinite-Dimensional Spaces. Mathematics 2021, 9, 266. https:// doi.org/10.3390/math9030266

Received: 15 January 2021 Accepted: 28 January 2021 Published: 29 January 2021

Publisher's Note: MDPI stays neutral with regard to jurisdictional clai$\mathrm{ms}$ in published maps and institutional affiliations.

Copyright: (C) 2021 by the authors. Licensee MDPI, Basel, Switzerland. This article is an open access article distributed under the terms and conditions of the Creative Commons Attribution (CC BY) license (https:// creativecommons.org/licenses/by/ $4.0 /)$.
Department of Applied Mathematics, University Politehnica of Bucharest, 060042 Bucharest, Romania; savin.treanta@upb.ro

Abstract: A new class of differential variational inequalities (DVIs), governed by a variational inequality and an evolution equation formulated in infinite-dimensional spaces, is investigated in this paper. More precisely, based on Browder's result, optimal control theory, measurability of set-valued mappings and the theory of semigroups, we establish that the solution set of DVI is nonempty and compact. In addition, the theoretical developments are accompanied by an application to differential Nash games.

Keywords: differential variational inequality; existence of solutions; bounded operator; evolutionary problem

\section{Introduction}

In the current paper, we introduce the following class of differential variational inequalities (DVIs) governed by a variational inequality and an evolution equation formulated in infinite-dimensional spaces:

$$
\begin{gathered}
x^{\prime}(\tau) \in \mathcal{A} x(\tau)+\mathcal{L} u(\tau)+H(\tau, x(\tau)), \quad \tau \in[0, T], \\
u(\tau) \in \mathcal{S}(\Omega, g(\tau, x(\tau))+\mathcal{F}(\cdot), \xi), \quad \text { a.e. } \tau \in[0, T], \\
x(0)=x_{0},
\end{gathered}
$$

where $\mathcal{S}(\Omega, g(\tau, x(\tau))+\mathcal{F}(\cdot), \xi)$ denotes the solution set of the following variational inequality (VI): find $u:[0, T] \rightarrow \Omega$ such that

$$
\langle g(\tau, x(\tau))+\mathcal{F}(u(\tau)), v-u(\tau)\rangle+\xi(v)-\xi(u(\tau)) \geq 0, \quad \forall v \in \Omega .
$$

Further, consider $\mathcal{X}$ and $\mathcal{X}_{1}$ as real infinite-dimensional Banach spaces, $\Omega \subset \mathcal{X}_{1}$ is a nonempty closed and convex subset, $\mathcal{A}: D(\mathcal{A}) \subset \mathcal{X} \rightarrow \mathcal{X}$ is the infinitesimal generator of a $C_{0}$-semigroup $e^{\mathcal{A} t}$ in $\mathcal{X}, \xi: \mathcal{X}_{1} \rightarrow(-\infty,+\infty]$ is a convex, lower semicontinuous, $\not \equiv+\infty$ functional, $\mathcal{L}: \mathcal{X}_{1} \rightarrow \mathcal{X}$ is a bounded linear operator, $H:[0, T] \times \mathcal{X} \rightarrow 2^{\mathcal{X}}$ is a set-valued mapping, $g:[0, T] \times \mathcal{X} \rightarrow \mathcal{X}_{1}^{*}$, and $\mathcal{F}: \Omega \rightarrow \mathcal{X}_{1}^{*}$ are given, which will be specified in Section 2.

In accordance with Pang and Stewart [1], Pazy [2] and Liu et al. [3], the solutions of evolutionary problem (DVI) are understood in the following mild sense:

Definition 1. A pair of functions $(x, u)$, with $x \in C([0, T] ; \mathcal{X})$ and $u:[0, T] \rightarrow \Omega$ measurable, is said to be a mild solution of evolutionary problem (DVI) if

$$
x(\tau)=e^{\mathcal{A} t} x_{0}+\int_{0}^{\tau} e^{\mathcal{A}(\tau-s)}[\mathcal{L} u(s)+h(s)] d s, \quad \tau \in[0, T],
$$

where $u(\tau) \in \mathcal{S}(\Omega, g(\tau, x(\tau))+\mathcal{F}(\cdot), \xi)$ and $h(\tau) \in H(\tau, x(\tau))$, a.e. $\tau \in[0, T]$. 
As is well-known, differential variational inequalities were introduced as a powerful mathematical tool of variational analysis in order to investigate real-life problems coming from operations research, engineering, and physical sciences. Various aspects related to differential variational inequalities have been investigated so far, but in a finitedimensional framework (see, for instance, [4-13] and references therein). The results established in Gwinner [6,7], Liu et al. [10], Pang and Stewart [1], Li et al. [14] are devoted to the case $\mathcal{X}=\mathbb{R}^{n}, \mathcal{X}_{1}=\mathbb{R}^{m}$ and $\mathcal{A}=0$. Recently, the theory of differential variational inequalities was extended to the more general level of infinite-dimensional Banach or Hilbert spaces. The paper by Liu et al. [15] was devoted to discuss the wellposedness and the generalized well-posedness of a differential mixed quasi-variational inequality and to provide criteria of well-posedness in the generalized sense of the inequality. For solving the problems or phenomena described by nonconvex superpotential functions, which are locally Lipschitz, Cen et al. [16] extended the results derived in Liu et al. [15]. Migórski and Bai [17] studied a class of evolution subdifferential inclusions involving history-dependent operators. Regarding the evolutionary problem (DVI), references Liu et al. $[3,18]$ could be seen as the most appropriate regarding the subject and the mathematical development presented in this paper. Reference Liu et al. [3] considers $\mathcal{L} u(\tau)+H(\tau, x(\tau))$ and $g(\tau, x(\tau))+\mathcal{F}(u(\tau))$ having the general form $f(\tau, x(\tau), u(\tau))$ and $g(\tau, x(\tau), u(\tau))$, respectively. By considering the particular form $g(\tau, x(\tau))+\mathcal{F}(u(\tau))$ for $g(\tau, x(\tau), u(\tau))$ is essential in the present paper since it allows us to use the Browder's theorem (see Theorem 1). On the other hand, the reference Liu et al. [18] does not contain the functional $\xi: \mathcal{X}_{1} \rightarrow(-\infty,+\infty]$ in the considered evolutionary problem. Thus, the evolutionary problem (DVI) becomes more general in this direction and incorporates various classes of problems and models.

In this paper, based on the Browder's theorem, optimal control theory, KKM theorem, measurability of set-valued mappings and the theory of semigroups, we study the existence of solutions associated with DVI in separable reflexive Banach spaces of infinite dimension. First, we prove important properties associated with the solution set of DVI as the stronglyweakly upper semicontinuity, superpositionally measurability, compactness and convexity. Further, we formulate and prove the main result established that the solution set for DVI is nonempty and compact. Additionally, the theoretical developments presented in this paper are accompanied by an application to differential Nash games. As is well known (see, for instance, Chen and Wang [4], Gwinner [6,7], Han and Pang [8], Li et al. [14], Liu et al. [10], Pang and Stewart [1], Wang and Huang [13]), this kind of evolutionary problem governed by variational inequalities includes various situations such as Coulomb friction problems for contacting bodies, dynamic traffic network, economical dynamics, electrical circuits with ideal diodes, control systems etc.

The paper is divided as follows. The first part of Section 2 includes basic definitions and results used further. Next, we establish a general existence result for a class of variational inequalities closely related to DVI. In addition, the strongly-weakly upper semicontinuity, compactness and superpositionally measurability are investigated for the solution set of the considered variational inequality. The final part of Section 2 provides some existence and qualitative properties for the solution set of the evolutionary problem (DVI). In order to illustrate the effectiveness of the theoretical results presented in this paper, an application to differential Nash games is also provided.

\section{Main Results}

In this section, we study the existence of solutions for DVI in infinite-dimensional Banach spaces, and we also formulate some properties of the solution set. First, we recall some notations, notions and results which will be useful in the sequel.

Let $2^{S}$ be the collection of all nonempty subsets for any nonempty set $S$. Additionally, we introduce

$$
\begin{gathered}
\Omega(S):=\left\{D \in 2^{S}: D \text { is compact }\right\} \\
\Omega_{v}(S):=\left\{D \in 2^{S}: D \text { is compact and convex }\right\}
\end{gathered}
$$


and denote by " $\rightarrow$ " and " $\rightarrow$ " the strong convergence and the weak convergence, respectively, in a given Banach space $\mathcal{X}$.

Definition 2 (see [19]). The set-valued mapping $F:[0, T] \rightarrow 2^{\mathcal{X}}$ is said to be measurable if the set $\{\tau \in[0, T]: F(\tau) \subset O\}$ is measurable on $\mathbb{R}$, for every open subset $O \subset \mathcal{X}$.

Definition 3. Let $\mathcal{X}$ and $\mathcal{X}_{1}$ be Banach spaces and let $I \subset R$ be an interval. The set-valued mapping $U: I \times \mathcal{X} \rightarrow 2^{\mathcal{X}_{1}}$ is superpositionally measurable if $\Phi: I \rightarrow 2^{\mathcal{X}_{1}}, \Phi(\tau)=U(\tau, x(\tau))$ is measurable, for every measurable set-valued mapping $x: I \rightarrow 2^{\mathcal{X}}$.

Lemma 1 (see [5]). Let $\mathcal{X}_{1}$ be a Hausdorff topological vector space, $\Omega \subset \mathcal{X}_{1}$ is a nonempty subset and $G: \Omega \rightarrow 2^{\mathcal{X}_{1}}$ is a set-valued mapping such that

(i) for any $\left\{v_{1}, \cdots, v_{n}\right\} \subset \Omega$, one has that its convex hull co $\left\{v_{1}, \cdots, v_{n}\right\}$ is included in $\bigcup_{i=1}^{n} G\left(v_{i}\right)$ (i.e., $G$ is a KKM mapping);

(ii) $G(v)$ is closed in $\mathcal{X}_{1}$ for every $v \in \Omega$;

(iii) $G\left(v_{0}\right)$ is compact in $\mathcal{X}_{1}$ for some $v_{0} \in \Omega$.

Then it holds $\bigcap_{v \in \Omega} G(v) \neq \varnothing$.

Lemma 2 (see [20]). $U: I \times \mathcal{X} \rightarrow \Omega\left(\mathcal{X}_{1}\right)$ is superpositionally measurable if it satisfies the Carathéodory condition or $U$ is upper or lower semicontinuous.

Then, the next theorem represents the first main result of this paper.

Theorem 1. Let $\mathcal{X}_{1}$ be a reflexive Banach space, $\Omega$ be a nonempty closed and convex subset of $\mathcal{X}_{1}$ and assume that

(i) $\mathcal{F}: \Omega \rightarrow \mathcal{X}_{1}^{*}$ is monotone on $\Omega$, that is

$$
\langle\mathcal{F}(v)-\mathcal{F}(u), v-u\rangle \geq 0, \quad \forall u, v \in \Omega,
$$

and satisfies

$$
\left.\lim _{\lambda \rightarrow 0^{+}} \inf \langle\mathcal{F}(\lambda u+(1-\lambda) v)), v-u\right\rangle \leq\langle\mathcal{F}(v), v-u\rangle, \quad \forall u, v \in \Omega ;
$$

(ii) $\xi: \mathcal{X}_{1} \rightarrow(-\infty,+\infty]$ is lower semicontinuous, convex, $\not \equiv+\infty$;

(iii) there exist $u_{0} \in \Omega$ and an $r>0$ such that

$$
\left\langle\mathcal{F}(v), v-u_{0}\right\rangle+\xi(v)-\xi\left(u_{0}\right)>0, \quad \forall v \in \Omega,\|v\|_{\mathcal{X}_{1}}>r
$$

and satisfies

$$
\liminf _{v \in \Omega ;\|v\|_{\mathcal{X}_{1} \rightarrow \infty}} \frac{\left\langle\mathcal{F}(v), v-u_{0}\right\rangle+\xi(v)-\xi\left(u_{0}\right)}{\|v\|_{\mathcal{X}_{1}}}=+\infty
$$

if the set $\Omega$ is unbounded in $\mathcal{X}_{1}$.

Then, for each element $w \in \mathcal{X}_{1}^{*}$, there exists $u \in \Omega$ such that

$$
\langle w+\mathcal{F}(u), v-u\rangle+\xi(v)-\xi(u) \geq 0, \quad \forall v \in \Omega,
$$

if and only if, for each element $w \in \mathcal{X}_{1}^{*}$, there exists $u \in \Omega$ such that

$$
\langle w+\mathcal{F}(v), v-u\rangle+\xi(v)-\xi(u) \geq 0, \quad \forall v \in \Omega .
$$

In addition, the solution set associated with (3) is nonempty, convex and closed in $\mathcal{X}_{1}$.

Proof. If $u \in \Omega$ is a solution associated with (3), by using the monotonicity of $\mathcal{F}$, it follows that $u \in \Omega$ is also a solution of (4). Conversely, assume that $u \in \Omega$ is a solution of (4). 
Taking into account the convexity of the set $\Omega$, for all $\lambda \in(0,1)$ and all $v \in \Omega$, it results that $u_{\lambda}:=(1-\lambda) u+\lambda v \in \Omega$. In consequence, we have

$$
\left\langle w+\mathcal{F}\left(u_{\lambda}\right), u_{\lambda}-u\right\rangle+\xi\left(u_{\lambda}\right)-\xi(u) \geq 0,
$$

and, by assumption (ii), we get

$$
\left\langle w+\mathcal{F}\left(u_{\lambda}\right), v-u\right\rangle+\xi(v)-\xi(u) \geq 0 .
$$

Considering $\lambda \rightarrow 0^{+}$in the above inequality (see the second part of hypothesis (i)), we obtain that $u \in \Omega$ is also a solution of (3).

Further, in order to prove the other assertions of our theorem, we consider the following two cases:

Case 1. $\Omega$ is bounded in $\mathcal{X}_{1}$. Consider the set-valued mapping $G: \Omega \rightarrow 2^{\Omega}$ defined as

$$
G(v):=\{u \in \Omega:\langle w+\mathcal{F}(v), v-u\rangle+\xi(v)-\xi(u) \geq 0, \quad \forall v \in \Omega\} .
$$

It is of course immediate (from the above equivalent conclusion) that $v \in G(v)$, whenever $v \in \Omega$, and $G(v)$ is a convex set.

Now, let us show that $G(v)$ is weakly closed in $\mathcal{X}_{1}$, for all $v \in \Omega$. Let $u_{n} \subset G(v)$ be a sequence with $u_{n} \rightarrow u$ in $\mathcal{X}_{1}$. Therefore, we have

$$
\left\langle w+\mathcal{F}(v), v-u_{n}\right\rangle+\xi(v)-\xi\left(u_{n}\right) \geq 0, \quad \forall v \in \Omega .
$$

Passing to the limit as $n \rightarrow+\infty$ in the above inequality, by hypothesis (ii), we get

$$
\langle w+\mathcal{F}(v), v-u\rangle+\xi(v)-\xi(u) \geq 0, \quad \forall v \in \Omega,
$$

that is $u \in G(v)$.

Further, we prove that the set-valued mapping $G$ is a KKM mapping (see Lemma 1). Suppose, by contradiction, that there exists $\left\{v_{1}, \cdots, v_{n}\right\} \subset \Omega$ and $u_{0}=\sum_{i=1}^{n} \lambda_{i} v_{i}$, with $\lambda_{i} \in[0,1]$ and $\sum_{i=1}^{n} \lambda_{i}=1$, satisfying $u_{0} \notin \bigcup_{i=1}^{n} G\left(v_{i}\right)$, that is

$$
\left\langle w+\mathcal{F}\left(v_{i}\right), v_{i}-u_{0}\right\rangle+\xi\left(v_{i}\right)-\xi\left(u_{0}\right)<0, \quad \forall i \in\{1,2, \cdots, n\} .
$$

By using the monotonicity of $\mathcal{F}$, it follows

$$
\left\langle w+\mathcal{F}\left(u_{0}\right), v_{i}-u_{0}\right\rangle+\xi\left(v_{i}\right)-\xi\left(u_{0}\right)<0, \quad \forall i \in\{1,2, \cdots, n\},
$$

from which the contradiction arises

$$
\begin{gathered}
0=\left\langle w+\mathcal{F}\left(u_{0}\right), u_{0}-u_{0}\right\rangle+\xi\left(u_{0}\right)-\xi\left(u_{0}\right) \\
\leq \sum_{i=1}^{n} \lambda_{i}\left[\left\langle w+\mathcal{F}\left(u_{0}\right), v_{i}-u_{0}\right\rangle+\xi\left(v_{i}\right)-\xi\left(u_{0}\right)\right]<0 .
\end{gathered}
$$

By using the hypothesis, it follows that $\Omega$ is weakly compact in $\mathcal{X}_{1}$. Thus, for each $v \in \Omega, G(v)$ is weakly compact in $\mathcal{X}_{1}$. Now, by applying Lemma 1 , we obtain $\bigcap_{v \in \Omega} G(v) \neq \varnothing$, that is the solution set associated with (4) is nonempty, so the same is true for the solution set associated with (3).

Case 2. $\Omega$ is unbounded in $\mathcal{X}_{1}$. For every integer $n \geq 1$, consider the bounded, closed and convex subset of $\mathcal{X}_{1}$

$$
\Omega_{n}:=\left\{x \in \Omega:\left\|x-u_{0}\right\|_{\mathcal{X}_{1}} \leq n\right\}
$$


where $u_{0} \in \Omega$ is given in assumption (iii). In accordance with the previous case, we can find $u_{n} \in \Omega_{n}$ such that

$$
\left\langle w+\mathcal{F}\left(u_{n}\right), v-u_{n}\right\rangle+\xi(v)-\xi\left(u_{n}\right) \geq 0, \quad \forall v \in \Omega_{n} .
$$

In the following, let us show that there exists an integer $k \geq 1$ such that

$$
\left\|u_{k}-u_{0}\right\|_{\mathcal{X}_{1}}<k
$$

By contradiction, assume that $\left\|u_{n}-u_{0}\right\|_{\mathcal{X}_{1}}=n$, for every integer $n \geq 1$. Putting $v=u_{0}$ in (5), we get

$$
\left\langle w+\mathcal{F}\left(u_{n}\right), u_{n}-u_{0}\right\rangle+\xi\left(u_{n}\right)-\xi\left(u_{0}\right) \leq 0,
$$

which is a contradiction (see assumption (iii)), if $n$ is sufficiently large. Therefore, the claim in (6) is fulfilled. By (6), for $y \in \Omega$ and sufficiently small $\tau>0$, we have

$$
\left\|u_{k}+\tau\left(y-u_{k}\right)-u_{0}\right\|_{\mathcal{X}_{1}}<k .
$$

Next, set $v=u_{k}+\tau\left(y-u_{k}\right)$ and $n=k$ in (5). By hypothesis (ii), it follows

$$
\left\langle w+\mathcal{F}\left(u_{k}\right), y-u_{k}\right\rangle+\xi(y)-\xi\left(u_{k}\right) \geq 0,
$$

that is, $u=u_{k}$ is a solution of variational problem (3).

Further, by using the equivalence between variational problems (3) and (4) and assumption (ii), we conclude that the solution set for (3) is closed and convex in $\mathcal{X}_{1}$. The proof is now complete.

Remark 1. Theorem 1 extends some results derived in Liu and Zeng [21], Liu et al. [3] and it is based on the Browder's theorem (see [22]). An extension of Minty's technique is given by assertion (4), and a generalized coercivity condition is provided by assumption (iii).

Corollary 1. Let $\mathcal{X}_{1}$ be a real reflexive Banach space and $\Omega \subset \mathcal{X}_{1}$ a nonempty compact and convex subset of $\mathcal{X}_{1}$. Then, the conclusion of Theorem 1 is fulfilled if $\mathcal{F}: \Omega \rightarrow \mathcal{X}_{1}^{*}$ and $\xi: \mathcal{X}_{1} \rightarrow$ $(-\infty,+\infty]$ verify conditions (i), (ii) in Theorem 1.

In the following, denote by $\mathcal{S}(\Omega, w+\mathcal{F}(\cdot), \xi)$ the set of solutions associated with the variational inequality (3). Closely related to the evolutionary problem (DVI), by using of Theorem 1, we also establish the following two results.

Lemma 3. For each integer $n>0$, under the same hypotheses of Theorem 1, there exists a constant $M_{n}>0$ satisfying

$$
\|u\|_{\mathcal{X}_{1}} \leq M_{n}, \quad u \in \mathcal{S}(\Omega, w+\mathcal{F}(\cdot), \xi), \forall w \in \bar{B}\left(n, \mathcal{X}_{1}^{*}\right):=\left\{w \in \mathcal{X}_{1}^{*}:\|w\|_{\left.\mathcal{X}_{1}^{*} \leq n\right\}}\right.
$$

Proof. By reductio ad absurdum, we assume that there exists $N_{0}>0$ satisfying

$$
\sup _{w \in \bar{B}\left(N_{0}, \mathcal{X}_{1}^{*}\right)}\left\{\|u\|_{\mathcal{X}_{1}}: u \in \mathcal{S}(\Omega, w+\mathcal{F}(\cdot), \xi)\right\}=+\infty .
$$

In consequence, there exist $w_{k} \in \bar{B}\left(N_{0}, \mathcal{X}_{1}^{*}\right)$ and $u_{k} \in \mathcal{S}\left(\Omega, w_{k}+\mathcal{F}(\cdot), \xi\right)$ satisfying $\left\|u_{k}\right\|_{\mathcal{X}_{1}}>k$, for $k=1,2, \ldots$. By assumption (iii) of Theorem 1, it follows that there is a function $p: \mathbb{R}_{+} \rightarrow \mathbb{R}_{+}$, with $p(k) \rightarrow+\infty$ as $k \rightarrow+\infty$, and a constant $M>0$ such that for each $\|u\|_{\mathcal{X}_{1}}>M$, we have

$$
\left\langle\mathcal{F}(u), u-v_{0}\right\rangle+\xi(u)-\xi\left(v_{0}\right) \geq p\left(\|u\|_{\mathcal{X}_{1}}\right)\|u\|_{\mathcal{X}_{1},} \quad v_{0} \in \Omega .
$$


Therefore, for $k>M$, one has $\left\|u_{k}\right\|_{\mathcal{X}_{1}}>M$ and, as $k$ large enough,

$$
\begin{gathered}
\left\langle w_{k}+\mathcal{F}\left(u_{k}\right), v_{0}-u_{k}\right\rangle+\xi\left(v_{0}\right)-\xi\left(u_{k}\right) \leq\left[\left\|w_{k}\right\|_{\mathcal{X}_{1}^{*}}-p\left(\left\|u_{k}\right\| \mathcal{X}_{1}\right)\right]\left\|u_{k}\right\|_{\mathcal{X}_{1}} \\
+\left\|w_{k}\right\|_{\mathcal{X}_{1}^{*}}\left\|v_{0}\right\|_{\mathcal{X}_{1}} \leq\left[N_{0}-p\left(\left\|u_{k}\right\|_{\mathcal{X}_{1}}\right)\right]\left\|u_{k}\right\|_{\mathcal{X}_{1}}+N_{0}\left\|v_{0}\right\|_{\mathcal{X}_{1}}<0 .
\end{gathered}
$$

This is a contradiction, and the proof is complete.

Further, for $g:[0, T] \times \mathcal{X} \rightarrow \mathcal{X}_{1}^{*}$, we consider a set-valued mapping $Z:[0, T] \times \mathcal{X} \rightarrow 2^{\mathcal{X}_{1}}$ given by

$$
Z(\tau, x):=\{u \in \Omega:\langle g(\tau, x)+\mathcal{F}(u), v-u\rangle+\xi(v)-\xi(u) \geq 0, \forall v \in \Omega\} .
$$

Theorem 2. Under the same hypotheses of Theorem 1, if $g:[0, T] \times \mathcal{X} \rightarrow \mathcal{X}_{1}^{*}$ is a continuous and uniformly bounded function, then

(i) the set-valued mapping $Z$ is strongly-weakly upper semicontinuous;

(ii) there exists $\psi>0$ satisfying

$$
\|Z(\tau, x(\tau))\|:=\sup _{u \in Z(\tau, x(\tau))}\|u\|_{\mathcal{X}_{1} \leq \psi, \quad \forall \tau \in[0, T],}
$$

for all $x \in C([0, T] ; \mathcal{X})$;

(iii) the set-valued mapping $\mathrm{Z}$ is superpositionally measurable.

Proof. (i) In accordance with Kamemsloo et al. [19], for each weakly closed subset $C$ of $\mathcal{X}_{1}$, we prove that $Z^{-}(C):=\{(\tau, x) \in[0, T] \times \mathcal{X}: Z(\tau, x) \cap C \neq \varnothing\}$ is strongly closed in $[0, T] \times \mathcal{X}$. Let prove that, if the sequence $\left(t_{n}, x_{n}\right) \in Z^{-}(C)$ and $\left(t_{n}, x_{n}\right) \rightarrow(\tau, x)$, then $(\tau, x) \in Z^{-}(C)$. By $\left(t_{n}, x_{n}\right) \in Z^{-}(C)$, for $n \in \mathbb{N}$, we obtain that there exists $u_{n} \in$

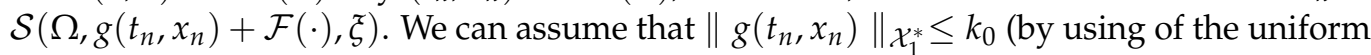
boundedness of $g$ ), where $k_{0}$ is a positive constant. According to Lemma 3, there exists a constant $M_{k_{0}}>0$ such that $\left\|u_{n}\right\|_{\mathcal{X}_{1}} \leq M_{k_{0}}$, for $n \in \mathbb{N}$. Consequently, the sequence $\left\{u_{n}\right\}$ is relatively weakly compact in $\mathcal{X}_{1}$, and we may assume $u_{n} \rightarrow u$, without loss of generality. Since $u_{n} \in \mathcal{S}\left(\Omega, g\left(t_{n}, x_{n}\right)+\mathcal{F}(\cdot), \xi\right)$, for $n \in \mathbb{N}$, we get

$$
\left\langle g\left(t_{n}, x_{n}\right)+\mathcal{F}\left(u_{n}\right), v-u_{n}\right\rangle+\xi(v)-\xi\left(u_{n}\right) \geq 0, \forall v \in \Omega .
$$

In virtue of the monotonicity of $\mathcal{F}$, it follows

$$
\left\langle g\left(t_{n}, x_{n}\right)+\mathcal{F}(v), v-u_{n}\right\rangle+\xi(v)-\xi\left(u_{n}\right) \geq 0, \forall v \in \Omega .
$$

Applying assumption (ii) of Theorem 1, the continuity of $g$ and $n \rightarrow \infty$, we have

$$
\langle g(\tau, x)+\mathcal{F}(v), v-u\rangle+\xi(v)-\xi(u) \geq 0, \forall v \in \Omega,
$$

that is, $u \in \mathcal{S}(\Omega, g(\tau, x)+\mathcal{F}(\cdot), \xi)$ (since the problem (4) is equivalent with the problem (3)). By using the weak closedness of $C$, we obtain $u \in Z(\tau, x) \cap C$. Therefore, $Z$ is strongly-weakly upper semicontinuous.

(ii) By hypothesis, $g:[0, T] \times \mathcal{X} \rightarrow \mathcal{X}_{1}^{*}$ is a continuous and uniformly bounded function. Therefore, for any $x \in C([0, T] ; \mathcal{X})$ and for all $\tau \in[0, T],\|g(\tau, x(\tau))\|_{\mathcal{X}_{1}^{*}}$ is uniformly bounded. By Lemma 3 , there exists $\psi$ satisfying

$$
\|Z(\tau, x(\tau))\|:=\sup _{u \in Z(\tau, x(\tau))}\|u\|_{\mathcal{X}_{1} \leq \psi, \quad \forall \tau \in[0, T] .}
$$

(iii) Since $Z$ is strongly-weakly upper semicontinuous with weakly compact convex values, then assertion (iii) holds true (see Lemma 2). The proof is complete.

In the following, consider the set-valued mapping $H:[0, T] \times \mathcal{X} \rightarrow \Omega_{v}(\mathcal{X})$ such that 
(a) for each $x \in \mathcal{X}$, the set-valued mapping $H(\cdot, x):[0, T] \rightarrow \Omega_{v}(\mathcal{X})$ is measurable;

(b) for a.e. $\tau \in[0, T]$, the set-valued mapping $H(\tau, \cdot): \mathcal{X} \rightarrow \Omega_{v}(\mathcal{X})$ is upper semicontinuous;

(c) for a.e. $\tau \in[0, T]$ and for all $x \in \mathcal{X}$, there exists $\rho \in L^{2}([0, T])$ satisfying

$$
\|h(\tau)\|_{\mathcal{X}} \leq \rho(\tau)\left(1+\|x(\tau)\|_{\mathcal{X}}\right), \quad \forall h(\tau) \in H(\tau, x(\tau)) .
$$

Taking into account the above assumptions for $H$, in accordance with Kamemsloo et al. [19], the superposition set-valued mapping $P_{H}: C([0, T] ; \mathcal{X}) \rightarrow 2^{L^{2}([0, T] ; \mathcal{X})}$, given by

$$
P_{H}(x):=\left\{h \in L^{2}([0, T] ; \mathcal{X}): h(\tau) \in H(\tau, x(\tau)), \text { a.e. } \tau \in[0, T]\right\}
$$

is well-defined.

The next theorem represents the central result of this paper. It investigates the existence of solutions for DVI.

Theorem 3. Let $e^{\mathcal{A t}}$ be a compact $C_{0}$-semigroup, $\mathcal{L}: \mathcal{X}_{1} \rightarrow \mathcal{X}$ a bounded linear operator and the assumptions (a)-(c) for $H$ be fulfilled. Under the hypotheses of Theorem 2, DVI has at least one mild solution $(x, u)$.

Proof. According to Pazy [2], for $u \in L^{2}\left([0, T] ; \mathcal{X}_{1}\right)$, the mild solutions of

$$
\begin{gathered}
x^{\prime}(\tau) \in \mathcal{A} x(\tau)+\mathcal{L} u(\tau)+H(\tau, x(\tau)), \quad \tau \in[0, T], \\
x(0)=x_{0}
\end{gathered}
$$

may denoted by

$$
x(\tau)=e^{\mathcal{A} t} x_{0}+\int_{0}^{\tau} e^{\mathcal{A}(\tau-s)}[\mathcal{L} u(s)+h(s)] d s, \quad \tau \in[0, T], h \in P_{H}(x) .
$$

By hypothesis and Rykaczewski [23] it follows that (7) is solvable, that is, there exists $x$ satisfying (8).

For every $\tau \in[0, T]$ and $M_{\mathcal{A}}:=\max _{\tau \in[0, T]}\left\|e^{\mathcal{A} t}\right\|$, from (8), we have the following estimates

$$
\begin{gathered}
\|x(\tau)\|_{\mathcal{X}} \leq\left\|e^{\mathcal{A} t} x_{0}\right\|_{\mathcal{X}}+\int_{0}^{\tau}\left\|e^{\mathcal{A}(\tau-s)}[\mathcal{L} u(s)+h(s)]\right\|_{\mathcal{X}} d s \\
\leq M_{\mathcal{A}}\left(\left\|x_{0}\right\|_{\mathcal{X}}+\|\mathcal{L}\|\|u\|_{L^{2}\left([0, T] ; \mathcal{X}_{1}\right)} T^{\frac{1}{2}}\right)+M_{\mathcal{A}} \int_{0}^{\tau} \rho(s)\left(1+\|x(s)\|_{\mathcal{X}}\right) d s,
\end{gathered}
$$

or, equivalently, by the Gronwall's inequality,

$$
\|x(\tau)\|_{\mathcal{X}} \leq M_{\mathcal{A}}\left(\left\|x_{0}\right\|_{\mathcal{X}}+\|\mathcal{L}\|\|u\|_{L^{2}\left([0, T] ; \mathcal{X}_{1}\right)} T^{\frac{1}{2}}+\|\rho\|_{L^{2}([0, T])}\right) e^{M_{\mathcal{A}}\|\rho\|_{L^{2}([0, T])}} .
$$

Next, we prove the existence of solutions for DVI. Set

$$
u_{k}(\tau)=\sum_{i=0}^{k-1} u^{(i)} \chi_{\left[\frac{i T}{k}, \frac{(i+1) T}{k}\right)}(\tau) \in Z\left(\frac{i T}{k}, x_{k}\left(\frac{i T}{k}\right)\right), \quad \tau \in\left[\frac{i T}{k}, \frac{(i+1) T}{k}\right), 0 \leq i \leq k-1,
$$

where $[0, T]=\cup_{i=1}^{k-1}\left[\tau_{i}, \tau_{i+1}\right) \cup\{T\}$, with $\tau_{i}:=\frac{i T}{k}$, and $\chi_{\left[\frac{i T}{k}, \frac{(i+1) T}{k}\right)}$ is the character function of interval $\left[\frac{i T}{k}, \frac{(i+1) T}{k}\right)$. Now, there exists $x_{k}(\cdot)$ satisfying

$$
x_{k}(\tau)=e^{\mathcal{A} t} x_{0}+\int_{0}^{\tau} e^{\mathcal{A}(\tau-s)}\left[\mathcal{L} u_{k}(s)+h_{k}(s)\right] d s, \quad \tau \in[0, T], h_{k} \in P_{H}\left(x_{k}\right) .
$$


For the sequences $\left\{u_{k}\right\}$ (see Theorem 2) there is $r_{1}>0$ satisfying $\left\|u_{k}\right\|_{L^{2}\left([0, T] ; \mathcal{X}_{1}\right)} \leq r_{1}$. By the above computations, we get $\left\|x_{k}\right\|_{C([0, T] ; \mathcal{X})} \leq r_{0}$, where $r_{0}>0$ is a constant. Thus, by assumption (c) of $H$, it follows that there is $r_{2}>0$ satisfying $\left\|h_{k}\right\|_{L^{2}([0, T] ; \mathcal{X})} \leq r_{2}$. Further, we may assume that $u_{k} \rightarrow u^{*}$ in $L^{2}\left([0, T] ; \mathcal{X}_{1}\right)$, without loss of generality, and $h_{k} \rightarrow h^{*}$ in $L^{2}([0, T] ; \mathcal{X})$. By using that $e^{\mathcal{A} t}$ is a compact $C_{0}$-semigroup and according to Li et al. [24], it results that $x_{k} \rightarrow x^{*}$ in $C([0, T] ; \mathcal{X})$, with

$$
x^{*}(\tau):=e^{\mathcal{A} t} x_{0}+\int_{0}^{\tau} e^{\mathcal{A}(\tau-s)}\left[\mathcal{L} u^{*}(s)+h^{*}(s)\right] d s .
$$

Further, by applying Mazur theorem (see Li et al. [14]), we obtain that there are $a_{i l} \geq 0, b_{i l} \geq 0$ with $\sum_{i \geq 1} a_{i l}=\sum_{i \geq 1} b_{i l}=1$ such that

$$
\begin{aligned}
& u_{l}(\tau):=\sum_{i \geq 1} a_{i l} u_{i+l}(\tau) \rightarrow u^{*}(\tau) \text { in } L^{2}([0, T] ; \mathcal{X}), \text { a.e. } \tau \in[0, T], \\
& h_{l}(\tau):=\sum_{i \geq 1} b_{i l} h_{i+l}(\tau) \rightarrow h^{*}(\tau) \text { in } L^{2}([0, T] ; \mathcal{X}), \text { a.e. } \tau \in[0, T] .
\end{aligned}
$$

Since $H(\tau, \cdot)$ is upper semicontinuous, $x_{k} \rightarrow x^{*}$ in $C([0, T] ; \mathcal{X})$, then for $\epsilon>0$ and $k>0$ large enough, we get

$$
H\left(\tau, x_{k}(\tau)\right) \subset H\left(\tau, x^{*}(\tau)\right)+B_{\epsilon},
$$

where $B_{\epsilon}$ is a ball in $\mathcal{X}$ centered in origin and radius $\epsilon$. Due to the convexity of $H\left(\tau, x^{*}(\tau)\right)+$ $B_{\epsilon}$, it follows that $h_{l}(\tau) \in H\left(\tau, x^{*}(\tau)\right)+B_{\epsilon}$, a.e. $\tau \in[0, T]$. Since $h_{l}(\tau) \rightarrow h^{*}(\tau)$ as $l \rightarrow \infty$, we get $h^{*}(\tau) \in \overline{H\left(\tau, x^{*}(\tau)\right)+B_{\epsilon}}$. For $\epsilon>0$ arbitrarly, it results $h^{*}(\tau) \in \overline{H\left(\tau, x^{*}(\tau)\right)}=$ $H\left(\tau, x^{*}(\tau)\right)$, a.e. $\tau \in[0, T]$. By the same arguments, we get $u^{*}(\tau) \in Z\left(\tau, x^{*}(\tau)\right)$, a.e. $\tau \in$ $[0, T]$. Consequently, there are $x^{*} \in C([0, T] ; \mathcal{X})$ and $u^{*} \in L^{2}\left([0, T] ; \mathcal{X}_{1}\right)$ such that

$$
x^{*}(\tau):=e^{\mathcal{A} t} x_{0}+\int_{0}^{\tau} e^{\mathcal{A}(\tau-s)}\left[\mathcal{L} u^{*}(s)+h^{*}(s)\right] d s, \quad \tau \in[0, T],
$$

where $u^{*}(\tau) \in Z\left(\tau, x^{*}(\tau)\right)$, a.e. $\tau \in[0, T]$ and $h^{*} \in P_{H}\left(x^{*}\right)$. The proof is complete.

Illustrative application. Consider a noncooperative differential Nash game with $N$ players. Let $\left(x^{\mu}, u^{\mu}\right) \in \mathcal{X}^{\mu} \times \mathcal{X}_{1}^{\mu}$ be the pair of state and control variables associated with player $\mu$, and $\Omega^{\mu} \subset \mathcal{X}_{1}^{\mu}$ be the nonempty, closed and convex set of admissible controls for player $\mu$. In addition, consider $x=\left(x^{\mu}\right)_{\mu=1}^{N} \in \mathcal{X}$ and $u=\left(u^{\mu}\right)_{\mu=1}^{N} \in \mathcal{X}_{1}$ be the collection of all players' variables, where

$$
\mathcal{X}=\prod_{\mu=1}^{N} \mathcal{X}^{\mu}, \quad \mathcal{X}_{1}=\prod_{\mu=1}^{N} \mathcal{X}_{1}^{\mu}
$$

For $\mu=\overline{1, N}$, consider $\Psi_{\mu}: \mathcal{X} \rightarrow \mathbb{R}, \phi_{\mu}:[0, T] \times \mathcal{X} \times \mathcal{X}_{1} \rightarrow \mathbb{R}$ are continuously differentiable functions, and introduce the following cost functional associated with player $\mu$,

$$
\Theta_{\mu}(x, u)=\Psi_{\mu}(x(T))+\int_{0}^{T} \phi_{\mu}(\tau, x(\tau), u(\tau)) d \tau .
$$

The corresponding optimization problem for player $\mu$ is to find an optimal trajetory $\left(x^{\mu}, u^{\mu}\right) \in \mathcal{X}^{\mu} \times \mathcal{X}_{1}^{\mu}$, for each fixed but arbitrary rival players' strategies $\left(x^{-\mu}, u^{-\mu}\right) \in$ $\mathcal{X}^{\mu} \times \mathcal{X}_{1}^{\mu}$, such that

$$
\text { Minimize } \Theta_{\mu}(x, u)
$$

subject to

$$
\begin{gathered}
\dot{x}^{\mu}(\tau)=q^{\mu}\left(\tau, x^{\mu}(\tau), u^{\mu}(\tau)\right), \\
x^{\mu}(0)=x_{0}^{\mu}, \quad u^{\mu}(\tau) \in \Omega^{\mu} \subset \mathcal{X}_{1}^{\mu},
\end{gathered}
$$


for almost all $\tau \in[0, T]$, where $q^{\mu}$ are continuously differentiable.

A differential Nash solution for the above optimization problem is a pair $\left(x^{*}, u^{*}\right) \in$ $\mathcal{X} \times \mathcal{X}_{1}$ such that for every $\mu=1, \cdots, N$, the pair $\left(x^{*}, \mu, u^{*, \mu}\right) \in \mathcal{X}^{\mu} \times \mathcal{X}_{1}^{\mu}$ solves player $\mu^{\prime} \mathrm{s}$ problem, given that $\mu^{\prime}$ s rivals all play their Nash strategies $\left(x^{*,-\mu}, u^{*,-\mu}\right) \in \mathcal{X}^{\mu} \times \mathcal{X}_{1}^{\mu}$.

Further, by using the Hamiltonian function associated with player $\mu$, with $\lambda^{\mu}$ the adjoint variable of player $\mu$,

$$
H_{\mu}\left(\tau, x, u, \lambda^{\mu}\right)=\phi_{\mu}(\tau, x, u)+\left(\lambda^{\mu}\right)^{t} q^{\mu}\left(\tau, x^{\mu}, u^{\mu}\right),
$$

we are able to formulate the necessary optimality conditions of first-order, as follows

$$
\begin{gathered}
\dot{\lambda}^{\mu}(\tau)=-\frac{\partial H_{\mu}}{\partial x^{\mu}}\left(\tau, x(\tau), u(\tau), \lambda^{\mu}(\tau)\right), \\
\dot{x}^{\mu}(\tau)=q^{\mu}\left(\tau, x^{\mu}(\tau), u^{\mu}(\tau)\right), \\
u^{\mu}(\tau) \in \mathcal{S}\left(\Omega^{\mu}, \frac{\partial H_{\mu}}{\partial u^{\mu}}\left(\tau, x(\tau), x^{-\mu}(\tau), \cdot, \lambda^{\mu}(\tau)\right),\right. \\
x^{\mu}(0)=x_{0}^{\mu}, \quad \lambda^{\mu}(T)=\frac{\partial \Psi_{\mu}}{\partial x^{\mu}}(x(T)) .
\end{gathered}
$$

In consequence, the study of a noncooperative differential Nash game with $N$ players is reduced to the study of the previous problem, which belongs to the class of problems investigated in the present paper.

\section{Conclusions}

In the current paper, based on Browder's result, optimal control theory, measurability of set-valued mappings and the theory of semigroups, we have investigated a new class of differential variational inequalities. More precisely, we have proved that the solution set associated with the considered evolutionary problem is nonempty and compact. In addition, the theoretical developments have been accompanied by an application to differential Nash games.

Funding: The APC was funded by University Politehnica of Bucharest, "PubArt" program.

Institutional Review Board Statement: Not applicable.

Informed Consent Statement: Not applicable.

Data Availability Statement: Not applicable.

Acknowledgments: The author would like to thank anonymous referees for their careful reading and constructive suggestions that substantially improved the revision of the manuscript.

Conflicts of Interest: The author declares no conflict of interest.

\section{References}

1. Pang, J.S.; Stewart, D.E. Differential variational inequalities. Math. Program. 2008, 113, 345-424. [CrossRef]

2. Pazy, A. Semigroups of Linear Operators and Applications to Partial Differential Equations; Springer: New York, NY, USA, 1983.

3. Liu, Z.H.; Zeng, S.D.; Motreanu, D. Evolutionary problems driven by variational inequalities. J. Differ. Equ. 2016, 260, 6787-6799. [CrossRef]

4. Chen, X.J.; Wang, Z.Y. Differential variational inequality approach to dynamic games with shared constraints. Math. Program. 2014, 146, 379-408. [CrossRef]

5. Fan, K. Some properties of convex sets related to fixed point theorems. Math. Ann. 1984, 266, 519-537. [CrossRef]

6. Gwinner, J. On the $p$-version approximation in the boundary element method for a variational inequality of the second kind modelling unilateral contact and given friction. Appl. Numer. Math. 2009, 59, 2774-2784. [CrossRef]

7. Gwinner, J. On a new class of differential variational inequalities and a stability result. Math. Program. Ser. B 2013, 139, $205-221$. [CrossRef]

8. Han, L.; Pang, J.S. Non-Zenoness of a class of differential quasi-variational inequalities. Math. Program. 2010, 121, 171-199. [CrossRef] 
9. Ke, T.D.; Loi, N.V.; Obukhovskii, V. Decay solutions for a class of fractional differential variational inequalities. Fract. Calc. Appl. Anal. 2015, 18, 531-553.

10. Liu, Z.H.; Loi, N.V.; Obukhovskii, V. Existence and global bifurcation of periodic solutions to a class of differential variational inequalities. Int. J. Bifurc. Chaos 2013, 23, 1350125. [CrossRef]

11. Loi, N.V. On two-parameter global bifurcation of periodic solutions to a class of differential variational inequalities. Nonlinear Anal. 2015, 22, 83-99. [CrossRef]

12. Pang, J.S.; Stewart, D.E. Solution dependence on initial conditions in differential variational variational inequalities. Math. Program. 2009, 116, 429-460. [CrossRef]

13. Wang, X.; Huang, N.J. A class of differential vector variational inequalities in finite dimensional spaces. J. Optim. Theory Appl. 2014, 162 633-648. [CrossRef]

14. Li, X.S.; Huang, N.J.; O’Regan, D. Differential mixed variational inequalities in finite dimensional spaces. Nonlinear Anal. 2010, 72, 3875-3886. [CrossRef]

15. Liu, Z.H.; Motreanu, D.; Zeng, S.D. On the well-posedness of differential mixed quasi-variational inequalities. Topol. Method Nonlinear Anal. 2018, 51, 135-150. [CrossRef]

16. Cen, J.; Min, C.; Nguyen, V.T.; Tang, G. On the well-posedness of differential quasi-variational-hemivariational inequalities. Open Math. 2020, 18, 540-551. [CrossRef]

17. Migórski S.; Bai, Y. Well-posedness of history-dependent evolution inclusions with applications. Z. Angew. Math. Phys. 2019, 70, 114. [CrossRef]

18. Liu, Z.H.; Zeng, S.D. Differential variational inequalities in infinite Banach spaces. Acta Math. Sci. 2017, 37, 26-32. [CrossRef]

19. Kamemsloo, M.; Obukhovskii, V.; Zecca, P. Condensing Multivalued Maps and Semilinear Differential Inclusions in Banach Space; Water de Gruyter: Berlin, Germany, 2001.

20. Zeidler, E. Nonlinear Functional Analysis and Its Applications. II/B. Nonlinear Monotone Operators; Springer: New York, NY, USA, 1990.

21. Liu, Z.H.; Zeng, B. Existence results for a class of hemivariational inequalities involving the stable $(g, f, \alpha)$-quasimonotonicity. Topol. Methods Nonlinear Anal. 2016, 47, 195-217.

22. Browder, F.E. Nonlinear monotone operators and convex sets in Banach spaces. Bull. Am. Math. Soc. 1965, 71, 780-785.

23. Rykaczewski, K. Approximate controllability of differential inclusions in Hilbert spaces. Nonlinear Anal. 2012, 75, 2701-2712. [CrossRef]

24. Li, X.J.; Yong, J.M. Optimal Control Theory for infinite Dimensional Systems; Birkhäuser: Boster, CA, USA, 1995. 